\title{
ON PARABOLIC MEASURES AND SUBPARABOLIC FUNCTIONS

\author{
BY
}

JANG-MEI G. WU

\begin{abstract}
Let $D$ be a domain in $R_{x}^{n} \times R_{t}^{1}$ and $\partial_{p} D$ be the parabolic boundary of $D$. Suppose $\partial_{p} D$ is composed of two parts $B$ and $S: B$ is given locally by $t=\tau$ and $S$ is given locally by the graph of $x_{n}=$ $f\left(x_{1}, x_{2}, \ldots, x_{n-1}, t\right)$ where $f$ is Lip 1 with respect to the local space variables and Lip $\frac{1}{2}$ with respect to the universal time variable. Let $\sigma$ be the $n$-dimensional Hausdorff measure in $R^{n+1}$ and $\sigma^{\prime}$ be the $(n-1)$ dimensional Hausdorff measure in $R^{n}$. And let $\operatorname{dm}(E)=\operatorname{d\sigma }(E \cap B)+d \sigma^{\prime}$ $\times \operatorname{dt}(E \cap S)$ for $E \subseteq \partial_{p} D$.

We study (i) the relation between the parabolic measure on $\partial_{p} D$ and the measure $d m$ on $\partial_{p} D$ and (ii) the boundary behavior of subparabolic functions on $D$.
\end{abstract}

For a Lipschitz domain in $R^{n}$, the relation between the harmonic measure and the $(n-1)$-dimensional Hausdorff measure on the boundary has been studied by Dahlberg [2], who proves among other results, that these two measures have the same null sets. In the first part of this note, we study the analogous problem for heat equation and prove that if a set on the boundary of a Lipschitz domain in $R^{n+1} \equiv R_{x}^{n} \times R_{t}^{1}$ (properly adapted for heat equation) is of certain Lebesgue type measure zero then it is the union of a set with parabolic measure zero and a set with adjoint parabolic measure zero. The inverse result has not been obtained yet. ${ }^{1}$

In the second part of this note, we study the boundary limits of subparabolic functions in a Lipschitz domain $D$ in $R^{n+1}$ and prove that if $u$ is a subparabolic function in $D$ with positive parabolic majorant and $\mathcal{L}$ is a uniformly separated family of parabolically nontangential curves in $D$ (defined in §3) ending on $E \subseteq \partial D$, then $u$ has limit along every curve ending in $E \backslash F$, where $F$ is the union of a set with parabolic measure zero and a set with adjoint parabolic measure zero. The subharmonic version of the above

Received by the editors February 1, 1978.

AMS (MOS) subject classifications (1970). Primary 35K05; Secondary 31B25.

Key words and phrases. Lipschitz domain, heat equation, parabolic measure, parabolic function, subparabolic function, Green's function, Schauder estimates, Harnack inequality, maximum principle, Brownian trajectories.

'Since the submitting of this paper, Robert Kaufman and the author have been able to show by example that the Lebesgue measure $d t$, the parabolic measure and the adjoint parabolic measure on $\partial \Omega$ can be mutually singular, where $\Omega=\{(x, t): x>f(t)\}$, for some real-valued Lip $\frac{1}{2}$ function $f$ on $R$. (To appear in Compositio Mathematica.) 
problem was first studied by Littlewood [10] for disks and radial lines in $\boldsymbol{R}^{2}$; various generalizations have been given by Privalov, Tolsted, Arsove and Huber, Dahlberg [3], Rippon [13] and Wu [15], [16], among others. For positive harmonic functions or positive parabolic functions, stronger limiting results have been obtained. Hunt and Wheeden [7] prove that a positive harmonic function in a Lipschitz domain in $R^{n}$ has nontangential limits almost everywhere on the boundary with respect to the harmonic measure (hence with respect to Lebesgue measure by [2]). Similar theorem for positive parabolic functions is proved by Kemper [9].

The ideas of our proofs follow the Laplacian case, the differences occur because the heat operator is not selfadjoint and the Harnack's inequality has a time-lag.

The author wishes to thank Richard Hunt for his suggestion of the first problem, Burgess Davis and Robert Kaufman for their conversations on Brownian motions, and N. A. Watson and the referee for their comments.

1. Preliminaries. By a domain we mean a bounded connected open set in $R^{n+1} \equiv R_{x}^{n} \times R_{t}^{1}$. We denote by $\partial_{p} D$ the parabolic boundary of a domain $D$; i.e., $\partial_{p} D$ is the set of points on the boundary of $D$ which are accumulation points of an open curve in $D$ along which the $t$-coordinate decreases as the boundary point is approached. We call a domain $D$ regular if the Dirichlet problem for the heat equation is solvable in the Brelot-Perron-Wiener sense for any Borel integrable boundary function. For two points $(x, t)$ and $(y, s)$ in $R^{m} \times R^{1}$ we denote by $d(x, t ; y, s)$ the parabolic distance $\left(|x-y|^{2}+\right.$ $|t-s|)^{1 / 2}$.

We call $D$, a domain in $R^{n+1}$, Lipschitz, if there exists $M>0$ and for every $(y, s)$ in $\partial_{p} D$ there is an open right cylinder $L$ containing $(y, s)$ such that one of the following occurs:

(i) $L \cap \partial_{p} D=L \cap\{t=s\}$ and $L \cap D=L \cap\{t>s\}$,

(ii) there is a local space coordinate system $\left(x^{\prime}, x_{n}\right), x^{\prime}=\left(x_{1}, \ldots, x_{n-1}\right) \in$ $R^{n-1}$ and $x_{n} \in R$, with the $x_{n}$-axis parallel to the axis of $L$ and a function $x_{n}=f\left(x^{\prime}, t\right)$, Lip 1 with respect to $x^{\prime}$ and Lip $\frac{1}{2}$ with respect to $t$, so that

$$
\begin{gathered}
\|f\| \equiv \sup \left|f\left(x^{\prime}, t\right)-f\left(x_{0}^{\prime}, t_{0}\right)\right| / d\left(x^{\prime}, t ; x_{0}^{\prime}, t_{0}\right)<M, \\
L \cap \partial_{p} D \cap\{t>s\}=L \cap\left\{(x, t): x_{n}=f\left(x^{\prime}, t\right)\right\} \cap\{t>s\}
\end{gathered}
$$

and

$$
L \cap D \cap\{t>s\}=L \cap\left\{(x, t): x_{n}>f\left(x^{\prime}, t\right)\right\} \cap\{t>s\} .
$$

We denote by $B$ (bottom points) the points in $\partial_{p} D$ satisfying (i), by $S$ (side points) the points in $\partial_{p} D$ satisfying (ii) and by $\Lambda$ (corner points) $=\bar{B} \cap S$. By a theorem of Petrowski [12], a Lipschitz domain is regular. 
Let $H=\sum_{1}^{n} \partial^{2} / \partial x_{k}^{2}-\partial / \partial t$ be the heat operator and $H^{*}=\Sigma_{1}^{n} \partial^{2} / \partial x_{k}^{2}$ $+\partial / \partial t$ be the adjoint heat operator. The solutions of $H u=0$ and $H^{*} u=0$ are called parabolic functions and adjoint parabolic functions, respectively. Corresponding to the super-harmonic functions and subharmonic functions for the Laplace operator, we also have superparabolic and subparabolic functions for $H$. The name "parabolic function" is adapted from [4], other names like heat function, temperature function, caloric function are also commonly used for solution of $H u=0$. For the basic properties of these functions, see [4] and [14]. The function

$$
W(x, t ; y, s) \equiv \begin{cases}{[4 \pi(t-s)]^{-n / 2} \exp \left[-\frac{|x-y|^{2}}{4(t-s)}\right],} & \text { for } t>s, \\ 0, & \text { for } t<s,\end{cases}
$$

is a solution of $H u=0$ in $R^{n+1} \backslash(y, s)$ for each fixed $(y, s)$ and is called the fundamental solution of the heat equation. We denote by $G$ and $G^{*}$ the Green's function and adjoint Green's function for a domain $D$, respectively, and have $G(x, t ; y, s)=G^{*}(y, s ; x, t)$. For a regular domain $D$, we have $G(x, t ; y, s)=W(x, t ; y, s)-U(x, t ; y, s)$ where for each fixed $(y, s) \in D$, $U$ is the parabolic function in $D$ corresponding to the boundary function $W$ on $\left\{(x, t) \in \partial_{p} D\right\}$. A very fundamental estimate for $W$ derived directly from definition can be formulated as follows.

LEMMA 1.1. For fixed $(y, s)$, let $\gamma(a)$ be the level surface of $W(x, t ; y, s)$ taking the value $(4 \pi a)^{-n / 2}$. Then $\gamma(a)$ is smooth, convex and lies in the region $\left\{(x, t): 0<t-s<a,|x-y|^{2}<2 a n / e\right\}$. Moreover, there are constants $b_{i}$ depending on $n, 0<b_{i}<1(i=1,2,3)$ so that $\gamma(a)$ encloses the region $\{(x, t)$ : $\left.b_{1} a<t-s<b_{2} a,\left|x_{i}-y_{i}\right|^{2}<b_{3} a(1 \leqslant i \leqslant n)\right\}$ in its interior, and there is $a$ constant $C$ depending on $n$ only, such that $W(x, t ; y, s) \leqslant C d(x, t ; y, s)^{-n}$.

For Schauder estimates, Harnack's inequality and the maximum principle about parabolic functions, we refer readers to [6], [11] and [14], respectively.

For fixed $(X, T) \in D$, we denote by $D(X, T)$ the set of points in $D$ which can be connected to $(X, T)$ by a curve in $D$ with strictly increasing $t$ coordinate.

LEMMA 1.2. Suppose $u_{k}$ is a sequence of parabolic functions on a domain $D$ with $u_{1} \leqslant u_{2} \leqslant u_{3} \leqslant \cdots$ on $D$. If there exists $\left(x_{0}, t_{0}\right) \in D$ such that $\lim _{k \rightarrow \infty} u_{k}\left(x_{0}, t_{0}\right)<\infty$, then $u_{k}$ and $\partial u_{k} / \partial x_{i}(1 \leqslant i \leqslant n)$ converge uniformly on compact subsets of $D\left(x_{0}, t_{0}\right)$ to parabolic functions $u$ and $\partial u / \partial x_{i}(1<i<$ $n)$, respectively.

This lemma is a parabolic analogue of Harnack's theorem. With the aid of Schauder estimates and the explicit integral representation of a parabolic 
function on an $(n+1)$-dimensional rectangle with continuous extension to the closure [6, p. 89], we may obtain Lemma 1.2 by following the classical proof of Harnack's theorem.

2. Parabolic measures. Let $D$ be a domain in $R_{x}^{n} \times R_{t}^{1}$, Lipschitz in the sense of $\S 1$. For a fixed point $(X, T)$ in $D$ and a Borel set $E \subseteq \partial D$, the parabolic measure [adjoint parabolic measure] of $E$ at $(X, T)$, denoted by $\omega_{D}^{(X, T)}(E)\left[\omega_{D}^{*(X, T)}(E)\right]$, is defined to be the value at $(X, T)$ of the solution of the heat [adjoint heat] equation on $D$ with boundary value 1 on $E$ and 0 on $\partial D \backslash E$ in the Brelot-Perron-Wiener sense. When there is no confusion, we shall suppress the subscript $D$.

Let $\sigma$ be the $n$-dimensional Hausdorff measure in $R^{n+1}$ and $\sigma^{\prime}$ be the (n-1)-dimensional Hausdorff measure in $R_{x}^{n} \times\{t=\tau\}$; let $d m$ be the measure defined on the Borel sets of $\partial_{p} D$ such that $d m(E)=d \sigma(E \cap B)+$ $d \sigma^{\prime} \times d t(E \cap S)$. We note that $d m \ll d \sigma$ on $S$ but $d \sigma$ need not be absolutely continuous with respect to $d m$ on $S$. One can easily construct a Lipschitz domain $D$ and a subset $E \subseteq \partial_{p} D$ such that $d m(E)=0$ and $d \sigma(E)=\infty$.

For a fixed point $(X, T)$ in $D$, let $\partial_{p} D(X, T)$ be the points in $\partial D$ which can be connected to $(X, T)$ by a curve in $D$ having strictly increasing $t$ coordinate.

One can show by the maximum principle that $\omega_{D}^{(X, T)}\left(\partial D \backslash \partial_{p} D\right)=0$ for each $(X, T) \in D$. We have the following measure comparison theorem on $\partial_{p} D$.

THEOREM 1. Let $D$ be a Lipschitz domain in $R^{n+1}$. Then:

(1) for each $(X, T) \in D, m(\Lambda)=\omega^{(X, T)}(\Lambda)=0$,

(2) for each $(X, T) \in D, \omega^{(X, T)}$ and $m$ have the same null sets on $B$ $\cap \partial_{p} D(X, T)$,

(3) if $E \subseteq S$ and $m(E)=0$, we can write $E$ as $E_{1} \cup E_{2}$ such that $\omega^{(X, T)}\left(E_{1}\right)$ $=0$ and $\omega^{*(X, T)}\left(E_{2}\right)=0$ for each $(X, T) \in D$.

Before we give the proof, we shall consider domains of special kinds and fix some notations.

Let $M>1$ be fixed and $f$ be a real-valued function defined on $R_{x^{\prime}}^{n-1} \times R_{t}^{1}$ with $\|f\|<M$ and support $f \subseteq\left\{\left(x^{\prime}, t\right):|t|^{1 / 2},\left|x_{i}\right|<2(1<i<n-1)\right\}$. Let $\Omega_{0}$ be a convex region with $C^{\infty}$ boundary, containing $\left\{|t|^{1 / 2},\left|x_{i}\right|<10\right.$ $(1 \leqslant i \leqslant n-1)$ and $\left.0<x_{n}<10 n M\right\}$, contained in $\left\{|t|^{1 / 2},\left|x_{i}\right|<11(1<i\right.$ $\leqslant n-1)$ and $\left.0<x_{n}<10 n M\right\}$ and satisfying $\Omega_{0} \cap\{t=\tau\}=\left(\Omega_{0}+(0, \tau-\right.$ $s)) \cap\{t=\tau\}$ for $|\tau|^{1 / 2},|s|^{1 / 2}<10$. And we let $\Omega$ be the region bounded by $\partial \Omega_{0} \backslash\left\{\left(x^{\prime}, 0, t\right):|t|^{1 / 2},\left|x_{i}\right|<2 \quad(1 \leqslant i \leqslant n-1)\right\} \cup\left\{\left(x^{\prime}, f\left(x^{\prime}, t\right), t\right):|t|^{1 / 2}\right.$, $\left.\left|x_{i}\right|<2(1 \leqslant i \leqslant n-1)\right\}$ and we denote by $P$ the point with $x^{\prime}=0, x_{n}=5 n$ $M$ and $t=25$.

In this section, we shall use the word "constant", or c's and C's, to denote 
constants depending only on $n$ and $M$ which may vary from line to line. Constants depending on other variables will be marked.

We denote by $\Delta((y, s), r)$ the region $\{(x, t): d(x, t ; y, s)<r\}$ and if $(y, s) \in \partial \Omega$, we denote by $A((y, s), r)$ the set $\partial \Omega \cap\left\{(x, t):\left|x_{i}-y_{i}\right|,|t|^{1 / 2}<r\right.$ $\left.(1 \leqslant i \leqslant n-1),\left|x_{n}-y_{n}\right|<2 n M r\right\}$.

LEMMA 2.1. Let $g^{*}$ be the adjoint Green's function on $\Omega$ and $\Omega_{1}=\Omega \backslash$ $\Delta(P, 1)$. Then there is a constant $C$ such that if $(x, t) \in \Omega_{1}$ then $\left(\partial / \partial x_{n}\right) g^{*}(x, t ; P)+C \geqslant 0$.

Proof. Let $\left\{f_{k}\right\}$ be a decreasing sequence of $C^{\infty}$ functions on $\left\{|t|^{1 / 2}\right.$, $\left.\left|x_{i}\right|<3(1 \leqslant i \leqslant n-1)\right\}$ with supports in $\left\{|t|^{1 / 2},\left|x_{i}\right|<2(1<i<n-1)\right\}$ and converging to $f$. We exhaust $\Omega$ by subdomains $\Omega(k)$, with boundaries $\partial \Omega(k)=\partial_{1}(k) \cup \partial_{2}(k)$ where $\partial_{1}(k)=\partial \Omega \backslash\left\{(x, t):|t|^{1 / 2},\left|x_{i}\right|<3(1<i<n\right.$ $\left.-1), x_{n}=f\left(x^{\prime}, t\right)\right\}$, and $\partial_{2}(k)=$ the graph of $f_{k}$. We assume, as we may, that $P \in \Omega(k)$. Let $g_{k}^{*}(x, t)$ be $g_{k}^{*}(x, t ; P)$, the adjoint Green's function of $\Omega(k)$ with singularity at $P$. We observe that $\sup \left\{g_{k}^{*}(x, t):(x, t) \in \Omega(k) \backslash \Delta\left(P, \frac{1}{2}\right)\right\}$ are uniformly bounded and that $\left(\partial / \partial x_{n}\right) g_{k}^{*}(x, t)$ is continuous on $\bar{\Omega}(k) \backslash \Delta\left(P, \frac{1}{2}\right)$ because $\partial \Omega(k)$ is $C^{\infty}$. By Schauder estimates we conclude that $\left\{\left|\left(\partial / \partial x_{n}\right) g_{k}^{*}(x, t)\right|, k \geqslant 1\right\}$ are uniformly bounded on $\partial \Delta(P, 1)$ and $\partial_{1}(k)$. On $\partial_{2}(k),\left(\partial / \partial x_{n}\right) g^{*}(x, t)$ has nonnegative boundary values. Letting $k \rightarrow \infty$, we conclude Lemma 2.1 by the adjoint form of Lemma 1.2.

LEMMA 2.2. Let $\mathrm{g}$ be the Green's function on $\Omega$, let $\omega$ be the parabolic measure on $\partial \Omega$, and let $a>1$. Then there are constants $c, C, \mu$ and constants $c(a), \rho(Q)$, $\rho(a, Q)$ depending on $a$ and (or) $Q$ such that whenever $Q \in E_{0} \equiv\left\{\left(x^{\prime}, f\left(x^{\prime}, t\right)\right.\right.$, $\left.t):|t|^{1 / 2},\left|x_{i}\right|<1(1 \leqslant i \leqslant n-1)\right\}$ and $0<\rho<\rho(Q)$ we have

$$
C \rho^{n} g(P, Q+(0, \mu \rho, 0)) \geqslant \omega^{P}(A(Q, \rho))>c \rho^{n} g(P, Q+(0, \mu \rho, 0))
$$

and whenever $Q \in E_{0}$ and $0<\rho<\rho(a, Q)$ we have

$$
\omega^{P}(A(Q, \rho)) \geqslant c(a) \omega^{P}(A(Q, a \rho)) \text {. }
$$

Proof. We let $\mu=4 n M$ and observe that the parabolic distance from $Q+(0, \mu \rho, 0)$ to $\partial \Omega$ is greater than $\rho$ and $Q+\left(0, \mu \rho, 2 \rho^{2}\right) \in \Omega$ if $0<\rho<1$ and $Q \in\left\{\left(x^{\prime}, f\left(x^{\prime}, t\right), t\right):|t|^{1 / 2}<8\right\} \cap \partial \Omega$. We let $\Omega^{\prime}=\Omega \cap\left\{x_{n}<10 n M-\right.$ $\left.r,|t|^{1 / 2}<8\right\}$ where $r$ is a constant to be determined later and $\rho_{0}=$ $\min \{1, r / \mu\}$. For $0<\rho<\rho_{0}$, we let $F_{\rho}(x, t)=\left(x^{\prime}, x_{n}+\mu \rho, t+2 \rho^{2}\right)$. It is clear that $F_{\rho}\left(\Omega^{\prime}\right) \subseteq \Omega$ and the parabolic distances from $F_{\rho}(A(Q, \rho))$ to $\partial \Omega$ and to $\{t=s\}$ are greater than $c \rho$ if $Q=(y, s) \in E_{0}$ and $0<\rho<\rho_{0}$.

Suppose $Q=(y, s) \in E_{0}$ and $0<\rho<\rho_{0}$. We note that there is a constant $c_{1}\left(0<c_{1}<1\right)$ such that the level curve $\gamma\left(c_{1}^{2} \rho^{2}\right)$ of $W(x, t ; Q+(0, \mu \rho, 0))$ is completely in $\Omega$. We write $g$ as $W-u$, where $u$ is positive parabolic in $\Omega$, and 
have

$$
\begin{aligned}
\max \{u(x, t ; Q & +(0, \mu \rho, 0)):(x, t) \in \partial \Omega\} \\
& <\max \{W(x, t ; Q+(0, \mu \rho, 0)):(x, t) \in \partial \Omega\} \\
& <\left(4 \pi c_{1}^{2} \rho^{2}\right)^{-n / 2}=(4 \pi)^{-n / 2} c_{1}^{-n} \rho^{-n}
\end{aligned}
$$

Therefore by the maximum principle $u(x, t ; Q+(0, \mu \rho, 0))<$ $(4 \pi)^{-n / 2} c_{1}^{-n} \rho^{-n}$ on $\Omega$. By Lemma 1.1, there are constants $0<b_{1}, b_{2}, b_{3}<1$ such that

$$
\begin{aligned}
h=\{ & (x, t): b_{1} c_{1}^{2} \rho^{2} / 4<t-s<b_{2} c_{1}^{2} \rho^{2} / 4, \\
& \left.\left|x_{i}-y_{i}\right|^{2}<b_{3} c_{1}^{2} \rho^{2} / 4(1<i<n-1),\left|x_{n}-y_{n}-\mu \rho\right|^{2}<b_{3} c_{1}^{2} \rho^{2} / 4\right\}
\end{aligned}
$$

is completely in the interior of $\gamma\left(c_{1}^{2} \rho^{2} / 4\right)$. Hence, if $(x, t) \in h, g(x, t ; Q+$ $(0, \mu \rho, 0))>C \rho^{-n}$ for some constant $c$. There exist constants $c$ and $C$ so that the parabolic distance from $h$ to the boundary of $\Omega \cap\{t>s\}$ and the $t$-coordinate distance from $h$ to $F_{\rho}(A(Q, \rho))$ are greater than $c \rho$, and the parabolic distance from $h$ to $F_{\rho}(A(Q, \rho))$ is at most $C \rho$. By Harnack's inequality, $g(x, t ; Q+(0, \mu \rho, 0))>c \rho^{-n}$ for $(x, t) \in F_{\rho}(A(Q, \rho))$.

Let $\omega^{\prime}$ be the harmonic measure of $\Omega^{\prime}$. Applying the maximum principle on $F_{\rho}\left(\Omega^{\prime}\right)$, we have

$$
g(x, t ; Q+(0, \mu \rho, 0))>C \omega^{\prime F_{p}^{-1}(x, t)}(A(Q, \rho)) \rho^{-n}
$$

for $(x, t) \in F_{\rho}\left(\Omega^{\prime}\right)$; in particular

$$
g(P ; Q+(0, \mu \rho, 0)) \geqslant C \omega^{\prime F_{\rho}^{-1}(P)}(A(Q, \rho)) \rho^{-n} .
$$

Because $F_{\rho}^{-1}(P) \in \Delta(P, 1)$ if $0<\rho<\frac{1}{2}$, we obtain by Harnack's inequality that

$$
g(P ; Q+(0, \mu \rho, 0))>c \omega^{\prime P_{0}}(A(Q, \rho)) \rho^{-n},
$$

where $P_{0}$ is the point with $x^{\prime}=0, x_{n}=5 n M$ and $t=20$. By Lemma 2.3 in [8] or Lemma 2.3 in [9], we may choose $r$, depending on $n$ and $M$, sufficiently small so that

$$
\omega^{\prime P_{0}}(A(Q, \rho))>C \omega^{P_{0}}(A(Q, \rho))
$$

if $Q \in E_{0}, 0<\rho<\rho_{0}$. It is proved in [9, p. 253] that the Poisson kernel $K(x, t ; y, s)$ on $\Omega$ defined by $\lim _{\rho \rightarrow 0} \omega^{(x, t)}(A((y, s), \rho)) / \omega^{P}(A((y, s), \rho))$ is a positive continuous function of $(y, s) \in \partial_{\rho} \Omega$ if $(x, t)$ is fixed. Therefore $K\left(P_{0}\right.$; $Q)>C>0$ for $Q \in E_{0}$. Thus for $Q \in E_{0}$, there exists $\rho(Q)<\rho_{0}$ so that if $0<\rho<\rho(Q)$ then

$$
\omega^{P_{0}}(A(Q, \rho))>C \omega^{P}(A(Q, \rho)) .
$$


Combining (2.3), (2.4) and (2.5), we have

$$
g(P ; Q+(0, \mu \rho, 0)) \geqslant c \omega^{P}(A(Q, \rho)) \rho^{-n},
$$

which is the first inequality in (2.1).

Let $Y$ be $\Delta(Q+(0, \mu \rho, 0), \rho / 2)$ for $Q \in E_{0}$ and $0<\rho<\rho_{0}$. The parabolic distance from $Y$ to $\partial \Omega$ is at least $c \rho$. By Lemma 1.1 in [9] and Harnack's inequality we have $\omega^{(x, t)}(A(Q, \rho)) \geqslant C$ for $(x, t) \in \bar{Y}$. Because $g<W$, we have $g(x, t ; Q+(0, \mu \rho, 0)) \leqslant c \rho^{-n}$ for $(x, t) \in \partial Y$. Applying the maximum principle on $\Omega \backslash Y$, we conclude that

$$
g(P ; Q+(0, \mu \rho, 0))<C \rho^{-n} \omega^{P}(A(Q, \rho)) \text {. }
$$

This proves the second part of (2.1).

The proof of (2.2) is similar to that of the first one in (2.1). We omit the proof.

Before we give more lemmas, we quote a theorem by Kemper [9] and remark on a certain covering of $E_{0}$.

THEOREM A (KEMPER). Suppose $D$ is a Lipschitz domain in $R^{n+1}, u$ is a nonnegative parabolic function on $D$ and $(X, T)$ is a point in $D$. Then $u$ has finite parabolic nontangential limit at each point in $\partial D$ except for a set of zero $\omega_{D}^{(X, T)}$ measure.

REMARK. Let $\mathcal{T}$ be the family $\left\{A(Q, \rho), Q \in E_{0}, \rho>0\right\}$. Then $\mathcal{V}$ forms a Vitali covering of $E_{0}$. If $A(Q, \rho) \in \mathscr{V}$ then $d \sigma^{\prime} \times d t(A(Q, 2 \rho))<C d \sigma^{\prime} \times$ $d t(A(Q, \rho))$; moreover, if $A(Q, \rho)$ and $A\left(Q_{0}, \rho\right) \in \mathcal{V}$ and $A(Q, \rho) \cap A\left(Q_{0}, \rho\right)$ $\neq \varnothing$ then $A\left(Q_{0}, \rho\right) \subseteq A(Q, c \rho)$, where $c$ and $C$ are constants depending on $n$ only.

LEMMA 2.3. Let $P^{*}$ be any point in $\Omega \cap\{t<-1\}$ and $g^{*}$ be the adjoint Green's function in $\Omega$. Then $\left(\partial g^{*} / \partial x_{n}\right)(x, t ; P)$ has finite parabolic nontangential limit $\omega^{* P^{*}}$-almost everywhere on $E_{0}$ and the set on which the limit is zero is of $\omega^{P}$ measure zero.

Proof. Let $E^{\prime}$ be the subset of $E_{0}$ on which the parabolic nontangential limit does not exist. It follows from Lemma 2.1 and the adjoint form of Theorem A that $\omega^{* P^{*}}\left(E^{\prime}\right)=0$. From Lemma 2.2 and the Lipschitz property of $\partial \Omega$, we have

$$
\frac{\omega^{P}(A(Q, \rho))}{d \sigma^{\prime} \times d t(A(Q, \rho))}<C \frac{g^{*}(Q+(0, \mu \rho, 0) ; P)}{\rho}
$$

for $Q \in E_{0}$ and $0<\rho<\rho(Q)$. By the remark on $\mathcal{W}$ and a modified theorem of Besicovitch [1, Theorem 5], the set in $E_{0} \backslash E^{\prime}$ on which the right-hand side of (2.6) has limit zero as $\rho \rightarrow 0$ is of $\omega^{P}$ measure zero. This proves the lemma. 
Lemma 2.4. Let $P^{*}=(0,5 n M,-25)$. Suppose $E \subseteq E_{0}$ and $d \sigma^{\prime} \times d t(E)=$ 0 . Then $E$ is composed of two parts $E_{1}$ and $E_{2}$ so that $\omega^{P}\left(E_{1}\right)=0$ and $\omega^{* P^{*}}\left(E_{2}\right)=0$.

Proof. From (2.6) and Lemma 2.3, we have

$$
\limsup _{\rho \rightarrow 0} \frac{\omega^{P}(A(Q, \rho))}{d \sigma^{\prime} \times d t(A(Q, \rho))}<\infty
$$

on $E_{0} \backslash E_{3}$ where $E_{3} \subseteq E_{0}$ and $\omega^{* P^{*}}\left(E_{3}\right)=0$. By the remark on the covering $\mathcal{V}$ and some classical arguments, it follows that $\omega^{P}\left(E \backslash E_{3}\right)=0$. We let $E_{1}=E \backslash E_{3}$ and $E_{2}=E \cap E_{3}$ and complete the proof.

Let $\tilde{\Omega}$ be the region $\left\{(x, t):\left|x_{i}\right|<10(1<i<n), 0<t<100\right\}, \tilde{P}$ be the point with $x=0$ and $t=25$ and $\tilde{E}_{0}$ be $\left\{(x, 0):\left|x_{i}\right|<1(1<i<n)\right\}$. Let $\tilde{g}$ and $\tilde{g}^{*}$ be the Green's function and the adjoint Green's function for $\tilde{\Omega}$, and let $\tilde{\omega}$ be the parabolic measure on $\partial \tilde{\Omega}$ with respect to $\tilde{\Omega}$. Then we have the following lemma.

LEMMA 2.5. (i) There are positive constants $c$ and $C$ so that $c<\tilde{g}^{*}(x, t ; P)<$ $C$ for $(x, t) \in \tilde{E}_{0}$.

(ii) Let $\tilde{Q}=(y, 0) \in \tilde{E}_{0}$ and $\tilde{A}(\tilde{Q}, r)=\left\{(x, 0):\left|x_{i}-y_{i}\right|<r(1<i<n)\right\}$. Then, for small $r$,

$$
c \tilde{g}^{*}\left(\tilde{Q}+\left(0, r^{2}\right) ; \tilde{P}\right) \leqslant \frac{\tilde{\omega}^{P}(\tilde{A}(\tilde{Q}, r))}{\sigma(\tilde{A}(\tilde{Q}, r))} \leqslant C \tilde{g}^{*}\left(\tilde{Q}+\left(0, r^{2}\right) ; \tilde{P}\right) .
$$

(iii) Suppose $\tilde{E} \subseteq \tilde{E}_{0}$. Then $\sigma(\tilde{E})=0$ if and only if $\tilde{\omega}^{\tilde{P}}(\tilde{E})=0$.

Proof. (i) follows from the fact that $\tilde{g}^{*}(\cdot ; \tilde{P})$ is positive and continuous on $\tilde{E}_{0}$. The proof of (ii) is similar to that of Lemma 2.2 and is only simpler, we omit it. (iii) follows from (i), (ii) and standard argument.

LEMMA 2.6. Suppose $D_{1}$ and $D_{2}$ are two domains in $R^{n+1}$ regular with respect to the Dirichlet problem of heat equation and $D_{2} \subseteq D_{1}$. Let $\omega_{i}^{(X, T)}$ be the parabolic measure on $\partial D_{i}(i=1,2)$ with respect to a fixed point $(X, T)$ in $D_{2}$. Suppose $E$ is a closed set on $\partial_{p} D_{1} \cap \partial_{p} D_{2}$ and there is an open set $V$ satisfying $E \subseteq V$ and $V \cap D_{1}=V \cap D_{2} \subseteq D_{2}(X, T)$. Then $\omega_{1}^{(X, T)}(E)=0$ if and only if $\omega_{2}^{(X, T)}(E)=0$.

Proof. The "only if" part follows from the maximum principle. If $\omega_{1}^{(X, T)}(E)>0$, let $u$ be the parabolic function on $D_{2}$ with boundary value $\omega_{1}^{(y, s)}(E)$ for $(y, s) \in D_{1} \cap \partial_{p} D_{2}$ and 0 for $(y, s) \in \partial_{p} D_{2} \backslash D_{1}$. Then $\omega_{2}^{(y, s)}(E)$ $=\omega_{1}^{(y, s)}(E)-u(y, s)$ is not identically zero on $D_{2}$, otherwise $\omega_{1}^{(y, s)}(E)$ has boundary value zero on $\partial_{p} D_{1} \cap \partial_{p} D_{2}$ which is a contradiction to the fact $\omega_{1} \neq 0$. If $\omega_{2}^{(X, T)}(E)=0$, then $\omega_{2}^{(y, s)}(E)=0$ on $D_{2}(X, T)$ from the maximum 
principle and $\omega_{2}^{(y, s)}(E)=0$ on $D_{2}$ by the assumption on $E$. This contradicts the fact that $\omega_{2} \neq 0$. This proves the "if" part. The same argument appeared in $[9$, p. 361].

Proof of Theorem 1. First we note that $B$ has at most countably many components and each component $B_{0}$ of $B$ lies in a hyperplane $\{t=s\}$.

Let $(y, s) \in \Lambda$ and $L, f$ be the cylinder and Lipschitz function corresponding to $(y, s)$ in the definition of Lipschitz domain. From the properties of $L$ and $f$ we see that $L \cap\left\{(x, s): x_{n}=f\left(x^{\prime}, s\right)\right\} \subseteq S$ and $L \cap\left\{(x, s): x_{n}>\right.$ $\left.f\left(x^{\prime}, s\right)\right\} \subseteq B_{0}$ for some component $B_{0}$ of $B$. Thus $(y, s) \in \partial B_{0}$ and $\Lambda \subseteq$ $\cup \partial B_{0}$. Moreover, from the discussion above, $B_{0}$ is Lipschitz in $R^{n} \times\{t=s\}$ for some $s$. Hence $m\left(\partial B_{0}\right)=0$ and $m(\Lambda)=0$. We assume, as we may, that $T>s$ and obtain, from the integeral representation of positive parabolic function in the half space $\{t>s\}$, that $\omega_{\{t>s\}}^{(X, T)}(\Lambda)=0$. By the maximum principle, $\omega_{D}^{(X, T)}\left(\partial B_{0}\right)=0$. Hence $\omega_{D}^{(X, T)}(\Lambda)=0$. This completes the proof of part (1).

Suppose $E \subseteq S \backslash \Lambda$ and $m(E)=0$. By dividing $E$ into countably many parts and changing the scale $\left(x \rightarrow a x+x_{0}, t \rightarrow a^{2} t+t_{0}\right)$ if necessary, we may assume that there is a real valued function $f$ on $R_{x^{\prime}}^{n-1} \times R_{t}^{1}$ with $\|f\|<M$ and support $f \subseteq\left\{\left(x^{\prime}, t\right):|t|^{1 / 2},\left|x_{i}\right|<2(1<i<n-1)\right\}$ and there is a region $\Omega$ corresponding to $f$ as defined before such that $\Omega \subseteq D$ and $E \subseteq\left\{\left(x^{\prime}, f\left(x^{\prime}, t\right), t\right):|t|^{1 / 2},\left|x_{i}\right|<1\right\} \subseteq \partial \Omega \cap \partial D$. It follows from Lemma 2.4, Lemma 2.6 and its adjoint form and the maximum principle that $E$ is composed of two parts $E_{1}$ and $E_{2}$ such that $\omega_{D}^{(X, T)}\left(E_{1}\right)=0$ and $\omega^{*(X, T)}\left(E_{2}\right)=$ 0 for each $(X, T) \in D$. This proves part (3).

The proof of part (2) is similar to that of (3) only we use Lemmas 2.5, 2.6 and the maximum principle instead. We shall not give the details.

3. Subparabolic functions. Let $D$ be a domain in $R^{n+1}$ Lipschitz in the sense of $\S 1, E$ be a subset of $\partial_{p} D$ and $\mathcal{L}_{E}$ be a family of arcs lying in $D$, each $Q$ in $E$ being the end point of a unique arc $l_{Q}$ in $\mathcal{L}_{E}$. The family $\mathcal{L}_{E}$ is said to be uniformly separated if there is a constant $K$, such that

$$
d\left(Q_{1} ; Q_{2}\right) \leqslant K \operatorname{dist}\left(l_{Q_{1}} ; l_{Q_{2}}\right)
$$

whenever $Q_{1}$ and $Q_{2}$ are both in $E \cap S$ or both in $E \cap B$ and where $d$ and dist denote parabolic distances. This definition was introduced by Rippon [13] for Laplace equation and Euclidean distances and had been used implicitly in [15, Theorem 1].

We say $\Gamma$ is a parabolic cone with vertex $(y, s)$ if there are positive numbers $k_{1}, k_{2}$ and $k_{3}$ and there are local space coordinates so that

$$
\Gamma=\left\{(x, t): k_{1}>x_{n}-y_{n}>k_{2}\left|x^{\prime}-y^{\prime}\right|+k_{3}|t-s|^{1 / 2}\right\}
$$


or

$$
\Gamma=\left\{(x, t): k_{1}>t-s>k_{2}|x-y|^{2}\right\} .
$$

We call a curve $l$ in $D$ ending at $(y, s) \in \partial_{p} D$ parabolically nontangential to $\partial_{p} D$ if there is a parabolic cone $\Gamma$ in $D$ with vertex $(y, s)$ and a neighborhood $U$ of $(y, s)$ so that $l \cap U \subseteq l \cap \bar{\Gamma} \subseteq D \cup\{(y, s)\}$.

We shall say that a set $E \subseteq \partial D$ has $\omega$ measure zero [ $\omega^{*}$ measure zero] if $\omega_{D}^{(x, t)}(E)=0\left[\omega_{D}^{*(x, t)}(E)=0\right]$ for every $(x, t) \in D$ and write $\omega(E)=0\left[\omega^{*}(E)\right.$ $=0]$. We note that if $E \subseteq \partial_{p} D(X, T)$ and $\omega_{D}^{(X, T)}(E)=0$ then $\omega_{D}^{(x, t)}(E)=0$ for every $(x, t) \in D$ by the maximum principle. A similar fact is true for $\omega^{*}$.

Theorem 2. Let $D$ be a Lipschitz domain in $R^{n+1}, E \subseteq \partial_{p} D$ and $\complement_{E}$ be $a$ uniformly separated family of parabolically nontangential curves in $D$. Suppose $u$ is a subparabolic function in $D$ which has a nonnegative parabolic majorant in $D$. Then there are sets $E_{1}, E_{2} \subseteq E$ satisfying $E_{2} \subseteq S, \omega\left(E_{1}\right)=0$ and $\omega^{*}\left(E_{2}\right)=$ 0 such that for $Q \in E \backslash\left(E_{1} \cup E_{2}\right), \lim u(P)$ exists as $P \rightarrow Q$ along $l_{Q}$.

By the Riesz decomposition theorem for subparabolic functions [4] and Theorem A, to show Theorem 2 it is enough to show the following.

THeOREM $2^{\prime}$. Let $D$ be a Lipschitz domain in $R^{n+1}, E \subseteq \partial_{p} D$ and $\mathcal{L}_{E}$ be a uniformly separated family of parabolically nontangential curves in $D$. Suppose $w$ is a Green's potential on $D$ given by a mass distribution $\nu$ in $D$ and $w \neq \infty$. Then there are sets $E_{1}, E_{2} \subseteq E$ satisfying $E_{2} \subseteq S, \omega\left(E_{1}\right)=0$ and $\omega^{*}\left(E_{2}\right)=0$ such that for $Q \in E \backslash\left(E_{1} \cup E_{2}\right), \lim w(P)=0$ as $P \rightarrow Q$ along $l_{Q}$.

We assume, as we may by Theorem 1 , that $E \subseteq \partial_{p} D \backslash \Lambda$. And we may assume that (i) there is a real-valued function $f$ on $R_{x^{\prime}}^{n-1} \times R_{t}^{1}$ with $\|f\|<M$ and support $f \subseteq\left\{\left(x^{\prime}, t\right):|t|^{1 / 2},\left|x_{i}\right|<2(1<i<n-1)\right\}$ such that the region $\Omega$ corresponding to $f$ (defined in $\$ 2$ ) is in $D$ and

$$
\begin{aligned}
E & \subseteq\left\{\left(x^{\prime}, f\left(x^{\prime}, t\right), t\right):|t|^{1 / 2},\left|x_{i}\right|<\frac{1}{2}\right\} \\
& \subseteq\left\{\left(x^{\prime}, f\left(x^{\prime}, t\right), t\right):|t|^{1 / 2},\left|x_{i}\right|<1(1<i<n-1)\right\} \subseteq \partial \Omega \cap \partial D
\end{aligned}
$$

or (ii) after proper transformations the region $\Omega$ defined in $\$ 2$ is in $D$ and

$$
\begin{aligned}
E & \subseteq\left\{(x, 0):\left|x_{i}\right|<1(1<i<n)\right\} \\
& \subseteq\left\{(x, 0):\left|x_{i}\right|<2(1<i<n)\right\} \subseteq \partial \tilde{\Omega} \cap \partial D .
\end{aligned}
$$

Let $\nu$ be the mass distribution in Theorem $2^{\prime}$ and let

$$
\begin{array}{ll}
v(x, t)=\int_{\Omega} g(x, t ; y, x) d \nu(y, s) & \text { on } \Omega, \\
\tilde{v}(x, t)=\int_{\tilde{\Omega}} \tilde{g}(x, t ; y, s) d \nu(y, s) & \text { on } \tilde{\Omega},
\end{array}
$$


where $g$ and $\tilde{g}$ are Green's functions on $\Omega$ and $\tilde{\Omega}$, respectively. We note that $w-v$ and $w-\tilde{v}$ are positive parabolic on $\Omega$ and $\tilde{\Omega}$, respectively. By Theorem A, they have parabolic nontangential limit on $\partial \Omega \cap \partial D$ except for a set of $\omega_{\Omega}$ measure zero or on $\partial \tilde{\Omega} \cap \partial D$ except for a set of $\tilde{\omega}_{\tilde{\Omega}}$ measure zero. And the limits are actually zero $\omega_{\Omega}$-a.e. or $\tilde{\omega}_{\tilde{\Omega}}$-a.e. by argument similar to that in [16, Proposition]. It follows from Lemma 2.6, its adjoint form and the maximum principle that in order to show Theorem $2^{\prime}$ it is enough to prove the following lemma.

LEMMA 3.1. Under the assumption (i) or (ii) in the last paragraph, $v$ has limit zero along $l_{Q}$ for $Q \in E$ with an exceptional set $E_{1} \cup E_{2}$, where $\omega_{\Omega}\left(E_{1}\right)=0$ and $\omega_{\Omega}^{*}\left(E_{2}\right)=0$; or $\tilde{v}$ has limit zero along $l_{Q}$ for $Q \in E$ with an exceptional set $\tilde{E}_{1}$ with $\tilde{\omega}_{\tilde{\Omega}}\left(\tilde{E}_{1}\right)=0$.

First we study $v$ on $\Omega$. We recall that $\mu=4 n M$ and $\Delta((x, t), r)=\{(y, s)$ : $d(x, t ; y, s)<r\}$. For a point $(x, t) \in \Omega$ with $|t|^{1 / 2},\left|x_{i}\right|<2(1<i<n-1)$ we denote by $(\tilde{x}, t)$ the point with $\tilde{x}^{\prime}=x^{\prime}, \tilde{x}_{n}=f\left(x^{\prime}, t\right)$. For $q>0$, we define $R(q)$ to be the region $\left\{(x, t) \in \Omega: 0<x_{n}-\tilde{x}_{n}<q\right.$ and $|t|^{1 / 2},\left|x_{i}\right|<1(1<i$ $\leqslant n-1)\}$. If $(x, t)$ and $(y, s)$ are in $R(1)$, we denote $x_{n}-\tilde{x}_{n}, y_{n}-\tilde{y}_{n}$ and $d\left(x^{\prime}, t ; y^{\prime}, s\right)$ by $\xi, \eta$ and $\delta$, respectively. We recall that $P$ is the point with $x^{\prime}=0, x_{n}=5 n M$ and $t=25$ and assume as we may that $v(P)<\infty$. We denote by $Z$ the set $\left\{(\tilde{x}, t) \in E_{0} \equiv \partial R(1) \cap \partial \Omega\right.$ : $\left(\partial / \partial x_{n}\right) g^{*}(x, t ; P)$ has positive finite limit as $\left.x_{n} \rightarrow \tilde{x}_{n}\right\}$ and recall from Lemma 2.3 that $E_{0} \backslash Z$ is composed of a set with $\omega_{\Omega}$ measure zero and a set with $\omega_{\Omega}^{*}$ measure zero.

After dividing $E$ into countably many parts, we assume as we may that there exist $\varepsilon>0$ and four parabolic cones at origin, $\Gamma^{i}(i=1,2,3,4)$ so that $\bar{\Gamma}^{i} \subseteq \Gamma^{i+1} \cup\{(0,0)\}(i=1,2,3)$ and $l_{\tilde{z}, \tau} \cap\left\{x_{n}<\tilde{z}_{n}+\varepsilon\right\} \subseteq \Gamma^{1}+(\tilde{z}, \tau), \bar{\Gamma}^{4}$ $+(\tilde{z}, \tau) \subseteq \Omega \cup\{(\tilde{z}, \tau)\}$ for each $(\tilde{z}, \tau) \in E$. For $(x, t) \in l_{\tilde{z}, \tau}$ and $(\tilde{z}, \tau) \in E$, we let $b(x, t)$ be $\left(\Gamma^{3}+(\tilde{z}, \tau)\right) \cap\left\{(y, s): x_{n}-\tilde{z}_{n}<2\left(y_{n}-\tilde{z}_{n}\right)<3\left(x_{n}-\tilde{z}_{n}\right)\right\}$. Moreover we may find $\alpha, \beta>1$ depending on $n, M$ and $\Gamma^{i}(1<i<4)$ so that whenever $(x, t) \in l_{\tilde{z}, \tau}$ and $(y, s) \in b(x, t)$ we have $\xi<\beta \eta$ and

$$
A((\tilde{z}, \tau), \eta) \subseteq A((\tilde{y}, s), \alpha \eta) .
$$

From now on we use the word "constant", $c$ or $C$, to denote constants depending on $n, M, \mathfrak{L}_{E}$ and $\Gamma^{i}(1<i<4)$ only and constants depending on other variables will be marked.

Lemma 3.2. Suppose $(\tilde{z}, \tau) \in E \cap Z,(x, t) \in l_{\tilde{z}, \tau}$ and $(y, s) \in b(x, t)$. Then if $\xi$ is sufficiently small (depending on $(\tilde{z}, \tau))$, we have

$$
g(x, t ; y, s) \leqslant C_{\tilde{z}, \tau} g(P ; y, s) \xi^{-1} \delta^{-n} .
$$

Proof. Let $(z, \tau)$ be the point with $z^{\prime}=\tilde{z}^{\prime}$ and $z_{n}=\tilde{z}_{n}+\mu \eta$. From Lemma 1.1 , the hypothesis $(\tilde{z}, \tau) \in Z$ and the fact $\xi<\beta \eta$, we have 


$$
\begin{aligned}
g(x, t ; y, s) & <C d(x, t ; y, s)^{-n}<C_{\tilde{z}, \tau} g^{*}(z, \tau ; P) \eta^{-1} \delta^{-n} \\
& <C_{\tilde{z}, \tau} g^{*}(z, \tau ; P) \xi^{-1} \delta^{-n},
\end{aligned}
$$

for $(x, t)$ sufficiently close to $(\tilde{z}, \tau)$. From Lemma 2.2 and (3.2) we have, for $(x, t)$ sufficiently close to $(\tilde{z}, \tau)$, that

$$
\begin{aligned}
g^{*}(z, \tau ; P) & \leqslant C \omega^{P}(A((\tilde{z}, \tau), \eta)) \eta^{-n}<C \omega^{P}(A((\tilde{y}, s), \alpha \eta)) \eta^{-n} \\
& <C \omega^{P}\left(A\left((\tilde{y}, s), \frac{\eta}{\mu}\right)\right) \eta^{-n} \\
& <C g^{*}(y, s ; P) .
\end{aligned}
$$

Therefore (3.3) follows from (3.4) and (3.5).

For $(\tilde{z}, \tau) \in E,(x, t) \in l_{\tilde{z}, \tau}$ we define

$$
v_{1}(x, t)=\int_{b(x, t)} g(x, t ; y, s) d \nu(y, s)
$$

and

$$
v_{2}(x, t)=\int_{\Omega \backslash b(x, t)} g(x, t ; y, s) d \nu(y, s) .
$$

We need the following two lemmas.

LEMMA 3.3. There are two sets $E_{1}$ and $E_{2}$ satisfying $\omega_{\Omega}\left(E_{1}\right)=0$ and $\omega_{\Omega}^{*}\left(E_{2}\right)$ $=0$ so that $v_{1}$ has limit zero along $l_{\Omega}$ if $Q \in E \backslash\left(E_{1} \cup E_{2}\right)$.

LEMMA 3.4. For $\omega_{\Omega}$-almost every $Q \in E, v_{2}$ has limit zero along $l_{Q}$.

Proof of Lemma 3.3. For $(\tilde{z}, \tau) \in E$ and $q>0$ we let $\varepsilon(q)=$ $\int_{R(q)} g(P ; y, s) d \nu(y, s)$ and let $\phi((\tilde{z}, \tau), r)$ be $\int g(P ; y, s) d \nu(y, s)$ extended over $N((\tilde{z}, \tau), r)=R(q) \cap\left(\cup\left\{\Delta((x, t), r):(x, t) \in l_{\tilde{z}, \tau}\right\}\right)$. Let $E(q)$ be the set of $(\tilde{z}, \tau)$ on which

$$
\lim _{r \rightarrow 0} \frac{\phi((\tilde{z}, \tau), r)}{d \sigma^{\prime} \times d t(A((\tilde{z}, \tau), r))}<\sqrt{\varepsilon(q)} .
$$

Noticing that $N\left(\left(\tilde{z}_{0}, \tau_{0}\right), r_{0}\right)$ and $N((\tilde{z}, \tau), r)$ are disjoint whenever $A((\tilde{z}, \tau)$, $2 K r)$ and $A\left(\left(\tilde{z}_{0}, \tau_{0}\right), 2 K r_{0}\right)$ are disjoint, where $K$ is the constant in (3.1), we conclude by argument similar to that in [5, p. 210] and the remark on the covering $\mathcal{T}$ that the complement of $E(q)$ in $E$ is of $d \sigma^{\prime} \times d t$ measure less than $C \sqrt{\varepsilon(q)}$.

For $(\tilde{z}, \tau) \in E(q) \cap Z \cap E$ and $(x, t) \in l_{\tilde{z}, \tau}$ sufficiently close to $(\tilde{z}, \tau)$, we obtain

$$
v_{1}(x, t)<\int_{0}^{C \xi} C_{\tilde{z}, \tau} \xi^{-1} r^{-n} d \Phi((\tilde{z}, \tau), r)
$$


with the aid of Lemma 3.2. By integration and (3.6) we have $\lim \sup v_{1}(x, t) \leqslant C_{\tilde{z}, \tau} \sqrt{\varepsilon(q)}$ as $(x, t) \rightarrow(\tilde{z}, \tau)$ along $l_{\tilde{z}, \tau^{*}}$ Because $d \sigma^{\prime} \times d t(E \backslash E(q)) \leqslant C \sqrt{\varepsilon(q)}$ for small $q$ and $\varepsilon(q) \rightarrow 0$ as $q \rightarrow 0$, $v_{1}$ has limit zero along $l_{\tilde{z}, \tau}$ for $d \sigma^{\prime} \times d t$-almost every $(\tilde{z}, \tau)$ in $Z \cap E$. From the property of $Z$ and Theorem 1 we conclude Lemma 3.3.

Before we prove Lemma 3.4, we quote a result of Doob [4, Theorems 5.1 and 8.2].

TheOREM B (DOOB). Suppose $D$ is a domain in $R^{n+1}$ and $P$ is a fixed point in $D$. Then a parabolic Green's potential in $D$, which is not identically infinity, has limit zero along almost every Brownian trajectory from $P$ to $\partial D$.

Proof of Lemma 3.4. Let $F$ be the collection of points $(\tilde{z}, \tau)$ in $E$ where $v_{2}$ does not have limit along $l_{\tilde{z}, \tau}$ or the limit exists but is not zero. After dividing $F$ into countably many collections, we assume that there is a number $\boldsymbol{m}>0$ such that for each $(\tilde{z}, \tau) \in F$, we may find a sequence of points $\left\{\left(x^{j}, t^{j}\right)\right\}$ on $l_{\tilde{z}, \tau} \cap\left(\Gamma_{1}+(\tilde{z}, \tau)\right)$ approaching $(\tilde{z}, \tau)$ with $v_{2}\left(x^{j}, t^{j}\right)>m$. Corresponding to each $\left(x^{j}, t^{j}\right)$, we let $\left(y^{j}, s^{j}\right)$ be the point on $\partial \Gamma_{2}+(\tilde{z}, \tau)$ with $y^{j \prime}=\tilde{z}^{\prime}, y_{n}^{j}=x_{n}^{j}$ and $s^{j}>t^{j}$. We denote $d\left(y^{j}, s^{j} ; \tilde{z}, \tau\right)$ by $d_{j}$ and note that there is a constant $\gamma<1$ depending on $\Gamma^{i}(i=1,2,3,4)$ so that

$$
\Delta\left(\left(y^{j}, s^{j}\right), 4 \gamma d_{j}\right) \subseteq b\left(x^{j}, t^{j}\right) \backslash\left(\Gamma^{1}+(\tilde{z}, \tau)\right) .
$$

By Harnack's inequality, we see that

$$
\begin{gathered}
\int_{\Omega \backslash b\left(x^{j}, t^{j}\right)} g(x, t ; y, s) d \nu(y, s) \geqslant c v_{2}\left(x^{j}, t^{j}\right) \geqslant \mathrm{cm} \\
\text { for }(x, t) \in \Delta_{j} \equiv \Delta\left(\left(y^{j}, s^{j}\right), \gamma d_{j}\right) . \text { Thus, for }(x, t) \in \Delta_{j}, \\
v(x, t) \geq \mathrm{cm} .
\end{gathered}
$$

We shall prove in the next lemma that, conditional on hitting $(\tilde{z}, \tau)$, the probability that Brownian trajectories from $P$ to $\partial \Omega$ pass through $\bar{\Delta}_{j}$ has a positive lower bound $\lambda$ which is independent of $(\tilde{z}, \tau)$ and $j$. Therefore by the countable additivity the conditional probability that Brownian trajectories from $P$ to $\partial \Omega$ pass through infinitely many $\bar{\Delta}_{j}$ 's before they hit $(\tilde{z}, \tau)$ has lower bound $\lambda$. Hence the probability that Brownian trajectories from $P$ to $\partial \Omega$ hit infinitely many $\left\{\bar{\Delta}_{j, \tilde{z}, \tau}: 1 \leqslant j \leqslant \infty,(\tilde{z}, \tau) \in F\right\}$ is at least $\lambda \cdot \omega_{\Omega}^{P}(F)$. It follows from (3.7) and Theorem B that $\omega_{\Omega}^{P}(F)=0$. This proves Lemma 3.4.

The next lemma is probably known, while the proof could not be found in the literature.

LEMMA 3.5. Let $F$ and $\Delta_{j}$ be as defined in the last lemma and let $(\tilde{z}, \tau) \in F$. Conditional upon hitting $(\tilde{z}, \tau)$, the probability that Brownian trajectories from $P$ to $\partial \Omega$ pass through $\bar{\Delta}_{j}$ has a positive lower bound $\lambda$ independent of $(\tilde{z}, \tau)$ and $j$. 
Proof. We recall that $\Delta_{j}=\Delta\left(\left(y^{j}, s^{j}\right), \gamma d_{j}\right)$. Given $(\tilde{z}, \tau) \in F$ and $1<j<$ $\infty$, we construct a Lipschitz subdomain $\Theta=\Theta(\tilde{z}, \tau, j)$ of $\Omega$ whose boundary is composed of $\partial_{1}$ and $\partial_{2}$ :

$$
\begin{gathered}
\partial_{1}=\partial \Omega \backslash\left\{\left(x^{\prime}, f\left(x^{\prime}, t\right), t\right):\left|t-s^{j}\right|^{1 / 2},\left|x_{i}-y_{i}^{j}\right|<2 d_{j}(1<i<n-1)\right\}, \\
\partial_{2}=\left\{\left(x^{\prime}, f_{0}\left(x^{\prime}, t\right), t\right):\left|t-s^{j}\right|^{1 / 2},\left|x_{i}-y_{i}^{j}\right|<2 d_{j}(1<i<n-1)\right\}
\end{gathered}
$$

for some Lipschitz function $f_{0}$ which satisfies (i) $f_{0}>f$, (ii) $\left\|f_{0}\right\|<C M(C$ independent of $(\tilde{z}, \tau)$ and $j$ ) and (iii) if $d\left(y^{j^{\prime}}, s^{j} ; x^{\prime}, t\right)<\gamma d_{j} / 2$, then $\left(x^{\prime}, f_{0}\left(x^{\prime}, t\right), t\right)$ is on $\partial \Delta_{j}$ with $f_{0}\left(x^{\prime}, t\right)>y_{n}^{j}$.

We let $\omega, \bar{\omega}$ and $\overline{\bar{\omega}}$ be the parabolic measures on the boundaries of $\Omega$, $\Theta(\tilde{z}, \tau, j)$ and $\Omega \backslash \Delta_{j}$, respectively, and let $A_{r}$ be $A((\tilde{z}, \tau), r)$, a subset of $\partial \Omega$ as defined before. Then the conditional probability $p$ that Brownian trajectories from $P$ to $\partial \Omega$ pass through $\bar{\Delta}_{j}$ (conditioned on hitting $(\tilde{z}, \tau)$ ) is at least

$$
\liminf _{\varepsilon \rightarrow 0}\left[\inf _{Q \in \bar{\Delta}_{j}} \frac{\overline{\bar{\omega}}^{P}\left(\partial \Delta_{j}\right) \cdot \omega^{Q}\left(A_{\varepsilon}\right)}{\omega^{P}\left(A_{\varepsilon}\right)}\right] \text {. }
$$

Let $Q_{0}$ be the point on $\bar{\Delta}\left(\left(y^{j}, s^{j}\right), 2 \gamma d_{j}\right)$ with the smallest time coordinate. By Harnack's inequality and the maximum principle we have

$$
p>C \liminf _{\varepsilon \rightarrow 0} \frac{\overline{\bar{\omega}}^{P}\left(\partial \Delta_{j}\right) \omega Q_{0}\left(A_{\varepsilon}\right)}{\omega^{P}\left(A_{\varepsilon}\right)} \geqslant C \liminf _{\varepsilon \rightarrow 0} \frac{\bar{\omega}^{P}\left(\partial_{2} \cap \partial \Delta_{j}\right) \omega^{Q_{0}}\left(A_{\varepsilon}\right)}{\omega^{P}\left(A_{\varepsilon}\right)} .
$$

From (iii) of the properties of $f_{0}$ above we see that

$$
\partial_{2} \cap \partial \Delta_{j} \supseteq\left\{\left(x^{\prime}, f_{0}\left(x^{\prime}, t\right), t\right): d\left(y^{j^{\prime}}, s^{j} ; x^{\prime}, t\right)<\gamma d_{j} / 2\right\} .
$$

By Lemma 2.2 we have

$$
p \geqslant C \liminf _{\varepsilon \rightarrow 0} \frac{\bar{\omega}^{P}\left(\partial_{2}\right) \omega Q_{0}\left(A_{\varepsilon}\right)}{\omega^{P}\left(A_{\varepsilon}\right)}
$$

if $j$ is large. By the maximum principle we have

$$
p \geqslant C \liminf _{\varepsilon \rightarrow 0} \frac{\omega^{P}\left(A\left((\tilde{z}, \tau), \gamma d_{j}\right)\right) \omega^{Q_{0}}\left(A_{\varepsilon}\right)}{\omega^{P}\left(A_{\varepsilon}\right)} .
$$

Applying Lemma 1.4 in [9] and Harnack's inequality, we obtain

$$
\omega^{P}\left(A(\tilde{z}, \tau), \gamma d_{j}\right) \omega^{Q_{0}}\left(A_{\varepsilon}\right) \geqslant C \omega^{P}\left(A_{\varepsilon}\right)
$$

if $\varepsilon<\gamma d_{j}$. The lemma follows from (3.8) and (3.9).

Proof of Lemma 3.1. The assertion about $v$ follows from Lemmas 3.3 and 3.4. Following the same argument as in Lemmas 3.3 and 3.4 but using Lemma 
2.5 instead of Lemmas 2.2 and 2.4, we may obtain the assertion about $\tilde{v}$. We omit the details.

The proof of Theorem 2 is thus complete.

\section{REFERENCES}

1. A. S. Besicovitch, $A$ general form of the covering principle and relative differentiation of additive functions. II, Proc. Cambridge Philos. Soc. 42 (1946), 1-10.

2. B. E. J. Dahlberg, On estimates of harmonic measures, Arch. Rational Mech. Anal. 65 (1977), 275-288.

3. On the existence of radial boundary values for functions subharmonic in a Lipschitz domain, Indiana Univ. Math. J. 27 (1978), 515-526.

4. J. L. Doob, A probability approach to heat equation, Trans. Amer. Math. Soc. 80 (1955), 216-280.

5. N. Dunford and J. T. Schwartz, Linear operators. I, Wiley-Interscience, New York, 1957.

6. A. Friedman, Partial differential equations of parabolic type, Prentice-Hall, Englewood Cliffs, N. J., 1964.

7. R. A. Hunt and R. L. Wheeden, On the boundary values of harmonic functions, Trans. Amer. Math. Soc. 132 (1968), 307-322.

8. J. T. Kemper, Kernel functions and parabolic limits for the heat equation, Thesis, Rice University, Houston, Texas, 1970.

9. _ Temperatures in several variables: kernel functions, representations, and parabolic boundary values, Trans. Amer. Math. Soc. 167 (1972), 243-262.

10. J. E. Littlewood, On functions subharmonic in a circle. II, Proc. London Math. Soc. (2) 28 (1928), 383-394.

11. J. Moser, A Harnack inequality for parabolic differential equations, Comm. Pure Appl. Math. 17 (1964), 101-134.

12. I. G. Petrowski, Zur ersten Randwertaufgaben der Warmeleitungsgleichung, Compositio Math. 1 (1935), 383-419.

13. P. J. Rippon, On the boundary behavior of Green potentials (preprint).

14. N. A. Watson, Green functions, potentials and the Dirichlet problem for the heat equation, Proc. London Math. Soc. (3) 33 (1976), 251-298.

15. J.-M. Wu, Boundary limits of Green's potentials along curves, Studia Math. 60 (1976), 137-144.

16. __ On functions subharmonic in a Lipschitz domain, Proc. Amer. Math. Soc. 68 (1978), 309-316.

Department of Mathematics, PUrdue University, West Lafayette, Indiana 47907 